\title{
On asymptotic limits for the quantum Heisenberg model
}

\author{
Joseph G Conlon and Jan Philip Solovej \\ Department of Mathematics, University of Michigan, Ann Arbor, MI 48109, USA
}

Received 6 March 1990

\begin{abstract}
We discuss various asymptotic limits of the classical and quantum Heisenberg model. We give a new proof that the thermodynamic free energy of the quantum model converges to the free energy of the classical model in the limit of large spins. We also obtain Gaussian and free Bose gas limits for the classical and quantum models respectively.
\end{abstract}

\section{Introduction}

We are interested in the Heisenberg model on a lattice in three dimensions. For simplicity we shall assume our lattice is the integer lattice $Z^{3}$. Suppose $\Lambda$ is a cube. Then $|\Lambda|$ is the number of points of the lattice contained in $\Lambda$. Let $\boldsymbol{S}=\left(S_{x}, S_{y}, S_{z}\right)$ be a spin vector. For the classical Heisenberg model $S$ is a vector on the unit sphere, whence

$$
\boldsymbol{S}^{2}=S_{x}^{2}+S_{y}^{2}+S_{z}^{2}=1 .
$$

For the quantum model $\boldsymbol{S}$ is the angular momentum operator acting on an irreducible representation of the 'rotation group' $\mathrm{SU}(2)$ with spin $s$, where $2 s$ is a non-negative integer. Thus $\boldsymbol{S}$ satisfies the identity

$$
\boldsymbol{S}^{2}=S_{x}^{2}+S_{y}^{2}+S_{z}^{2}=s(s+1)
$$

and the commutation relations

$$
\left[S_{x}, S_{y}\right]=\mathrm{i} S_{z} \quad\left[S_{y}, S_{z}\right]=\mathrm{i} S_{x} \quad\left[S_{z}, S_{x}\right]=\mathrm{i} S_{y} .
$$

If we introduce the operators $S_{+}$and $S_{-}$given by

$$
S_{+}=S_{x}+\mathrm{i} S_{y} \quad S_{-}=S_{x}-\mathrm{i} S_{y}
$$

the relations (1.3) can be reformulated as

$$
\left[S_{z}, S_{+}\right]=S_{+} \quad\left[S_{z}, S_{-}\right]=-S_{-} \quad\left[S_{+}, S_{-}\right]=2 S_{z} .
$$

To define the Heisenberg model on a cube $\Lambda$ we associate to each site $R \in \Lambda$ a spin vector $\boldsymbol{S}(R)$. The Heisenberg model is then given by

$$
\dot{S}_{1}=-\sum_{R, R^{\prime}=1} J\left(R-R^{\prime}\right) \boldsymbol{S}(R) \cdot S\left(R^{\prime}\right)+h \sum_{R} S_{z}(R) .
$$

We shall assume from here on that $J(R)=J(-R) \geqslant 0, J(0)=0$ and $h \geqslant 0$. Hence we are considering a ferromagnetic system in which the ground state occurs when all spins are aligned along the negative $z$ axis. If we wish to specify the representatation for the quantum model we write $\mathfrak{S}_{\mathrm{i}, \mathrm{s}}$ and if we wish to specify the classical model we 
write $\tilde{S}_{1, c}$. The partition function for the quantum model will be denoted $Z_{\mathrm{s}}(\beta, h, \Lambda)$. Thus

$$
Z_{\mathrm{s}}(\beta, h, \Lambda)=\operatorname{Tr} \exp [-\beta \tilde{\mathcal{S}} \backslash, \mathrm{s}] .
$$

The classical partition function is denoted $Z_{\mathrm{c}}$ whence

$$
Z_{\mathrm{c}}(\beta, h, \Lambda)=\int \exp \left[-\beta \tilde{5}{ }_{1, c}\right] \prod_{R} \mathrm{~d} S(R)
$$

and the integration is taken over the unit sphere.

Our first goal here is to derive in a simple way a formula of Dyson [3] which identifies the partition function for the Heisenberg model with the partition function of an anharmonic oscillator. This representation has two advantages. In the first case it exhibits the magnon approximation explicitly in the oscillator Hamiltonian as the dominant term at large values of $\beta$. Secondly, it shows that the magnon-magnon interaction is a two-body interaction. We also obtain the classical analogue of Dyson's formula. Motivated by this we give a very simple proof that the free energy for the quantum Heisenberg model converges in the large-s limit to the free energy of the classical Heisenberg model. This result was originally proved by Lieb [6]. We also show that a large-s scaling limit of the quantum Heisenberg ferromagnet with nearestneighbour interaction converges to a free Bose gas. For the classical model the corresponding limit gives the Gaussian approximation. Finally we prove some magnontype bounds on the magnetisation for the Heisenberg model. This extends and simplifies some work of Roepstorff [9].

\section{Dyson's formula}

We first consider the quantum case. To do this we need to compute the commutator of $\tilde{S}_{1, s}$ with $S_{+}(W)$ where $W \in \Lambda$. Using the fact that, for $R \neq R^{\prime}$, we have

$$
S(R) \cdot S\left(R^{\prime}\right)=\frac{1}{2}\left(S_{-}(R) S_{-}\left(R^{\prime}\right)+S_{-}(R) S_{+}\left(R^{\prime}\right)\right)+S_{z}(R) S_{z}\left(R^{\prime}\right)
$$

and the commutation relations (1.5) we have

$\left[\tilde{S}_{1.5} S_{+}(W)\right]=2 \sum_{R} J(R-W)\left(S_{z}(W) S_{+}(R)-S_{z}(R) S_{+}(W)\right)+h S_{+}(W)$.

Commuting again with $S_{+}\left(W^{\prime}\right)$ we obtain the relation

$$
\begin{aligned}
& {\left[\left[\tilde{S}_{1, s}, S_{+}(W)\right], S_{+}\left(W^{\prime}\right)\right]} \\
& \quad=-2 J\left(W-W^{\prime}\right) S_{+}(W) S_{+}\left(W^{\prime}\right)+2 \delta_{W W} \sum_{R} J(R-W) S_{+}(W) S_{+}(R)
\end{aligned}
$$

where $\delta$ denotes the Kronecker $\delta$. It is clear from this that all higher-order commutators will vanish.

The basic idea of Dyson is to come up with a harmonic oscillator Hamiltonian which satisfies the same double-commutation relations as $\tilde{5}_{1, s}$. To accomplish this we assign an oscillator to each site $R$. Thus $a^{*}(R), a(R)$ are creation and annihilation operators satisfying canonical commutation relations $\left[a(R), a^{*}\left(R^{\prime}\right)\right]=\delta_{R R^{\prime}}$. We look for an oscillator Hamiltonian $5 \%$, which satisfies the commutation relation

$$
\begin{aligned}
& {\left[\left[\mathfrak{F}_{1, W}^{0}, a^{*}(W)\right], a^{*}\left(W^{\prime}\right)\right]} \\
& \quad=-2 J\left(W-W^{\prime}\right) a^{*}\left(W^{\prime}\right) a^{*}(W)+2 \delta_{W, w} \sum_{R} J(R-W) a^{*}(W) a^{*}(R) .
\end{aligned}
$$


It is evident that one solution to $(2.4)$ is

$\mathfrak{S Q}_{\mathrm{A}, \mathrm{s}}^{0}=-\sum_{R, R^{\prime} \in \mathrm{A}} J\left(R-R^{\prime}\right)\left[a^{*}(R) a(R) a^{*}\left(R^{\prime}\right) a\left(R^{\prime}\right)-a^{*}(R) a(R)^{2} a^{*}\left(R^{\prime}\right)\right]$.

The term in [ ] could of course have been written in a more symmetric way. We can add to this a constant plus a quadratic form in $a, a^{*}$ without changing the commutation relations (2.4). Thus we can take

$$
\begin{aligned}
\mathfrak{S P}_{\mathrm{i}, \mathrm{s}}^{0}=E_{\mathrm{1}, \mathrm{S}}+ & \sum_{R, R^{\prime} \in \mathrm{\Lambda}} a^{*}(R) A_{R, R^{\prime}} a\left(R^{\prime}\right)-\sum_{R, R^{\prime} \in \mathrm{\Lambda}} J\left(R-R^{\prime}\right) \\
& \times\left[a^{*}(R) a(R) a^{*}\left(R^{\prime}\right) a\left(R^{\prime}\right)-a^{*}(R) a(R)^{2} a^{*}\left(R^{\prime}\right)\right]
\end{aligned}
$$

where $E_{1, s}$ and $A_{R, R^{\prime}}$ are arbitrary constants.

Next we choose $E_{A, s}$ so that $\mathscr{S}_{\Lambda, s}^{0}$ acts on the vacuum in the same manner as $\mathscr{S}_{\Lambda, s}$ acts on its ground state. Let $|m\rangle_{R}$ for $0 \leqslant m \leqslant 2 s$ be the state for which $S_{z}(R)=m-s$. Correspondingly let $\mid m)_{R}$ be the $m$-particle state for $a^{*}(R) a(R)$. The ground state of $\tilde{S}_{1, \mathrm{~s}}$ is then

$$
\prod_{R \in A}|0\rangle_{R}
$$

and the vacuum for $\mathscr{S}_{\Lambda, s}^{0}$ is

$$
\left.\prod_{R \in \Lambda} \mid 0\right)_{R}
$$

It is clear then that if we choose $E_{1 . s}$ to be given as the ground state energy of $\mathscr{S}_{1, s}$,

$$
E_{\mathrm{A}, \mathrm{s}}=-\sum_{R, R^{\prime} \in \mathrm{\Lambda}} J\left(R-R^{\prime}\right) s^{2}-h \sum_{R \in \mathrm{\Lambda}} s
$$

then we have

$$
\begin{aligned}
& \mathfrak{S}_{\mathrm{\Lambda}, \mathrm{s}} \prod_{R \in \Lambda}|0\rangle_{R}=E_{\Lambda, \mathrm{s}} \prod_{R \in \Lambda}|0\rangle_{R} \\
& \left.\mathfrak{\mathcal { Y }}_{\Lambda, \mathrm{s}}^{0} \prod_{R \in \Lambda} \mid 0\right)_{R}=E_{\Lambda, \mathrm{s}} \prod_{R \in \Lambda}|0\rangle_{R} .
\end{aligned}
$$

It is clear from the above that $E_{\mathrm{A}, \mathrm{s}}$ has been chosen in a unique way. The matrix elements $A_{R, R^{\prime}}$ can also be chosen in a unique way by requiring that $\mathfrak{S}_{\mathrm{A}, \mathrm{s}}$ and $\mathfrak{S}_{\mathrm{A}, \mathrm{s}}^{0}$ behave in a similar way on one-particle states. Let $u=\left\{u_{R}: R \in \Lambda\right\}$ be a set of nonnegative integers $u_{R}=0,1,2, \ldots$ Then the state $\left.\mid u\right)$ is defined as

$$
\mid \boldsymbol{u})=\prod_{R \in \Lambda}\left(u_{R}\right)_{R}
$$

with a similar definition for $|\boldsymbol{u}\rangle$. The one-particle state $|R|$ is defined as $|R|=\mid \boldsymbol{u}$ ) where $u_{R}=1$ and $u_{R^{\prime}}=0$ when $R \neq R^{\prime}$. The action of the Heisenberg Hamiltonian on oneparticle states $|R\rangle$ is given by

$$
\tilde{S}_{\Lambda, \mathrm{s}}|R\rangle=\left(E_{\Lambda, \mathrm{s}}+h\right)|R\rangle+2 s \sum_{R^{\prime}} J\left(R-R^{\prime}\right)\left[|R\rangle-\left|R^{\prime}\right\rangle\right] .
$$

If we now choose $A_{R, R^{\prime}}$ to be given by

$$
A_{R, R^{\prime}}=\left(h+2 s \sum_{R^{\prime \prime}} J\left(R-R^{\prime \prime}\right)\right) \delta_{R R^{\prime}}-2 s J\left(R-R^{\prime}\right)
$$

it is evident that

$$
\left.\left.\left.\left.\mathfrak{S}_{\Lambda, \mathrm{s}}^{0} \mid R\right)=\left(E_{\Lambda, \mathrm{s}}+h\right) \mid R\right)+2 s \sum_{R^{\prime}} J\left(R-R^{\prime}\right)[\mid R)-\mid R^{\prime}\right)\right] .
$$


Finally we observe that because of the identity of the double-commutator relationships (2.3) and (2.4) and the vanishing of higher-order commutators it follows that if

$$
\left.\left.\dot{S}_{1, s}^{0} \mid \boldsymbol{u}\right)=\sum_{\boldsymbol{w}^{\prime}} \Gamma_{\boldsymbol{w}, \boldsymbol{u}} \mid \boldsymbol{w}\right)
$$

then we must also have

$$
\tilde{S}_{1, \mathbf{s}}|\boldsymbol{u}\rangle=\sum_{\boldsymbol{w}} \Gamma_{\boldsymbol{w}, \boldsymbol{u}}|\boldsymbol{w}\rangle \text {. }
$$

Note that equations (2.15) and (2.16) are independent of the normalisation of the states $\mid u),|u\rangle$. In $(2.16)$ we put $|\boldsymbol{u}\rangle=0$ if any $u_{R}$ exceeds $2 s$. It follows immediately from (2.15) and (2.16) that

$$
Z_{\mathrm{s}}(\beta, h, \Lambda)=\operatorname{Tr}_{\mathrm{s}} \exp \left[-\beta \tilde{5}_{\mathrm{i}}^{0}{ }_{\mathrm{s}}\right]
$$

where $\operatorname{Tr}_{s}$ denotes restricted trace in which one sums over states with particle number at most $2 s$ at each site. Thus

$$
\operatorname{Tr}_{\mathrm{\varsigma}}(\boldsymbol{A})=\sum_{\substack{0 \leqslant u_{R} \leq 2 s \\ R \in 1}}(\boldsymbol{u}|\boldsymbol{A}| \boldsymbol{u})
$$

The formula (2.17) is Dyson's formula [3]. The first two terms in the expression (2.6) for $\mathfrak{S}_{1,5}^{0}$ give the magnon approximation for the Heisenberg model.

We now move on to deriving the analogue of Dyson's formula for the classical Heisenberg model. First we shall motivate the formula from the quantum problem. Then we shall derive it directly. Let $F(\xi)$ be a real-valued function for $-\infty<\xi<\infty$. Thus $F$ is an 'observable'. From $(2.15),(2.16)$ it follows that

$$
\left\langle\boldsymbol{u}\left|F\left(\tilde{\boldsymbol{S}}_{\mathrm{i}, \mathrm{s}}\right)\right| \boldsymbol{u}\right\rangle=\left(\boldsymbol{u}\left|F\left(\tilde{S}_{1, \mathrm{~s}}^{0}\right)\right| \boldsymbol{u}\right)
$$

where $|\boldsymbol{u}\rangle$ and $\mid \boldsymbol{u})$ are normalised states. Another way of writing (2.19) is

$$
\begin{aligned}
& \text { average value of } F\left(\tilde{S}_{1, s}\right) \text { on } S_{z}(R)=u_{R}-s \quad R \in \Lambda \\
& \text { = average value of } F\left(\tilde{S}_{\text {. }}^{0} \text { ) on } a^{*}(R) a(R)=u_{R} \quad R \in \Lambda\right. \text {. }
\end{aligned}
$$

We want to turn (2.20) into a classical formula. To do this we scale the quantum Hamiltonian so that it converges to the classical Heisenberg Hamiltonian. Thus in the quantum Hamiltonian we replace the field $h$ by $s h$ and let $s$ be large. Then from $(2.20)$ we have

$$
\begin{array}{cc}
\text { average value of } F\left(\tilde{S}_{1, s} / s^{2}\right) \text { on } S_{z}(R) / s=u_{R} / s-1 & R \in \Lambda \\
=\text { average value of } F\left(\dot{S}_{1, s}^{0} / s^{2}\right) \text { on } a^{*}(R) a(R) / s=u_{R} / s & R \in \Lambda .
\end{array}
$$

Next we put

$$
\begin{aligned}
& a(R)=(s / 2)^{1 / 2}(q(R)+\mathrm{i} p(R)) \\
& a^{*}(R)=(s / 2)^{1 / 2}(q(R)-\mathrm{i} p(R))
\end{aligned}
$$

and let $s \rightarrow \infty$. Then we have

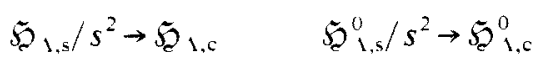

where $\tilde{S}_{1, c}^{0}$ is the classical Hamiltonian given by the expression

$$
\begin{aligned}
\mathfrak{S O}_{1.0}^{0}=E_{1.0} \sum_{R, R^{\prime}=1}(q(R)-\mathrm{i} p(R)) \\
\quad \times\left\{\left[h / 2+\sum_{R^{\prime \prime}} J\left(R-R^{\prime \prime}\right)\right] \delta_{R R^{\prime}}-J\left(R-R^{\prime}\right)\right\}\left(q\left(R^{\prime}\right)+\mathrm{i} p\left(R^{\prime}\right)\right) \\
\quad-\frac{1}{4} \sum_{R, R^{\prime}} J\left(R-R^{\prime}\right)\left[\left(q^{2}(R)+p^{2}(R)\right)\left(q^{2}\left(R^{\prime}\right)+p^{2}\left(R^{\prime}\right)\right)\right. \\
\left.-\left(q^{2}(R)+p^{2}(R)\right)(q(R)+\mathrm{i} p(R))\left(q\left(R^{\prime}\right)-\mathrm{i} p\left(R^{\prime}\right)\right)\right]
\end{aligned}
$$


where $E_{1, c}$ is the minimum energy of the classical Heisenberg Hamiltonian

$$
E_{\backslash, \mathrm{c}}=-\sum_{R, R^{\prime} \in \backslash} J\left(R-R^{\prime}\right)-h \sum_{R} 1 \text {. }
$$

The quantum identity (2.21) converges now as $s \rightarrow \infty$ to the classical identity

$$
\begin{aligned}
& \text { average value of } F\left(\dot{\mathcal{S}}_{1, \mathrm{c}}\right) \text { on } S_{z}(R)=u_{R}-1 \quad R \in \Lambda \\
= & \text { average value of } F\left(\dot{5}_{\mathrm{i}, \mathrm{c}}^{0}\right) \text { on } q^{2}(R)+p^{2}(R)=2 u_{R} \quad R \in \Lambda
\end{aligned}
$$

where $u_{R}$ is restricted to the interval $0 \leqslant u_{R} \leqslant 2$.

If for each $R$ we introduce angular variables $\theta(R), 0 \leqslant \theta(R) \leqslant 2 \pi$, which parametrise the circles $S_{z}(R)=u_{R}-1$ and $q^{2}(R)+p^{2}(R)=2 u_{R}$, then we may write (2.26) as

$$
\int F\left(\tilde{S}_{\uparrow, c}\right) \prod_{R} \mathrm{~d} \theta(R)=\int F\left(\tilde{\mathfrak{S}}_{\uparrow, c}^{0}\right) \prod_{R} \mathrm{~d} \theta(R) .
$$

If we integrate (2.27) with respect to $u_{R}$ over $0 \leqslant u_{R} \leqslant 2, R \in \Lambda$, and recall that

$$
\begin{aligned}
& \mathrm{d} \theta(R) \mathrm{d} u_{R}=\mathrm{d} \theta(R) \mathrm{d} S_{z}(R)=\mathrm{d} S(R) \\
& \mathrm{d} \theta(R) \mathrm{d} u_{R}=\mathrm{d} q(R) \mathrm{d} p(R)
\end{aligned}
$$

where $\mathrm{d} S(R)$ is the standard measure on the unit sphere, we obtain the equation

$$
\int F\left(\mathfrak{S}_{1 ., \mathrm{c}}\right) \prod_{R} \mathrm{~d} S(R)=\int F\left(\mathfrak{S}_{1, \mathrm{c}}^{0}\right) \prod_{R} \mathrm{~d} q(R) \mathrm{d} p(R)
$$

where the $\mathrm{d} q(R) \mathrm{d} p(R)$ integration is over the inside of the circle $q^{2}(R)+p^{2}(R) \leqslant 4$. If we take now $F(\xi)=\exp [-\beta \xi]$ we have

$$
\begin{aligned}
& Z_{\mathrm{c}}(\beta, h, \Lambda)=\int_{D} \exp \left[-\beta 5_{\mathrm{E}}^{0}{ }_{1, \mathrm{c}}\right] \prod_{R} \mathrm{~d} q(R) \mathrm{d} p(R) \\
& D=\prod_{R}\left[(q(R), p(R)) \mid q(R)^{2}+p\left(R^{2}\right) \leqslant 4\right] .
\end{aligned}
$$

Equation (2.30) is the classical analogue of Dyson's formula.

Equation (2.30) can be derived without reference to the quantum problem. To accomplish this we write the classical Heisenberg Hamiltonian (1.6) in the $u_{R}, \theta(R)$ variables, $R \in \Lambda$, by substituting

$$
S_{x}(R)+\mathrm{i} S_{y}(R)=\left(2 u_{R}-u_{R}^{2}\right)^{1 / 2} \exp [\mathrm{i} \theta(R)] \quad S_{z}(R)=u_{R}-1
$$

where $0 \leqslant u_{R} \leqslant 2$. Then we have

$$
\begin{gathered}
\mathfrak{S}_{\mathrm{A}, \mathrm{c}}=-\sum_{R, R^{\prime} \in \mathrm{I}} J\left(R-R^{\prime}\right)\left[\frac { 1 } { 2 } ( 2 u _ { R } - u _ { R } ^ { 2 } ) ^ { 1 / 2 } ( 2 u _ { R ^ { \prime } } - u _ { R ^ { \prime } } ^ { 2 } ) ^ { 1 / 2 } \left\{\exp \left[\mathrm{i}\left(\theta(R)-\theta\left(R^{\prime}\right)\right)\right]\right.\right. \\
\left.+\exp \left[\mathrm{i}\left(\theta\left(R^{\prime}\right)-\theta(R)\right)\right]\right\}+\left(u_{R}-1\right)\left(u_{R^{\prime}}-1\right) \rrbracket+h \sum_{R}\left(u_{R}-1\right) .
\end{gathered}
$$

We can in a similar fashion write $\hat{S}_{\mathrm{i}, \mathrm{c}}^{0}$ if we make the substitution

$$
q(R)+\mathrm{ip}(R)=\left(2 u_{R}\right)^{1 / 2} \exp [\mathrm{i} \theta(R)] .
$$

We obtain the expression

$$
\begin{aligned}
\mathfrak{S}_{A, \mathrm{c}}^{0}=E_{A, \mathrm{c}}+ & \sum_{R, R^{\prime} \in \Lambda}\left[\left(h / 2+\sum_{R^{\prime \prime}} J\left(R-R^{\prime \prime}\right)\right) \delta_{R R^{\prime}}-J\left(R-R^{\prime}\right)\right] 2\left(u_{R} u_{R^{\prime}}\right)^{1 / 2} \\
& \times \exp \left[\mathrm{i}\left(\theta(R)-\theta\left(R^{\prime}\right)\right)\right]-\sum_{R, R^{\prime} \in \Lambda} J\left(R-R^{\prime}\right)\left\{u_{R} u_{R^{\prime}}-u_{R}\left(u_{R} u_{R^{\prime}}\right)^{1 / 2}\right. \\
& \left.\times \exp \left[\mathrm{i}\left(\theta(R)-\theta\left(R^{\prime}\right)\right)\right]\right\} .
\end{aligned}
$$


We can put the expressions $(2.32),(2.34)$ in a more convenient form:

$$
\begin{aligned}
& \tilde{S}_{\mathrm{i}, \mathrm{c}}=E_{\mathrm{\backslash}, \mathrm{c}}+\sum_{R, R^{\prime} \in \mathrm{\backslash}} J\left(R-R^{\prime}\right)\left(2 u_{R}-u_{R} u_{R^{\prime}}\right)+h \sum_{R} u_{R} \\
& -\sum_{R, R^{\prime} \in 1} J\left(R-R^{\prime}\right)\left(u_{R} u_{R}\right)^{1 / 2}\left(2-u_{R}\right)^{1 / 2}\left(2-u_{R^{\prime}}\right)^{1 / 2} \\
& \times \exp \left[\mathrm{i}\left(\theta(R)-\theta\left(R^{\prime}\right)\right)\right] \\
& \tilde{S}_{1, \mathrm{c}}^{0}=E_{1, \mathrm{c}}+\sum_{R, R^{\prime} \in \mathrm{l}} J\left(R-R^{\prime}\right)\left(2 u_{R}-u_{R} u_{R^{\prime}}\right)+h \sum_{R} u_{R} \\
& -\sum_{R, R^{\prime}=1} J\left(R-R^{\prime}\right)\left(u_{R} u_{R^{\prime}}\right)^{1 / 2}\left(2-u_{R}\right) \exp \left[\mathrm{i}\left(\theta(R)-\theta\left(R^{\prime}\right)\right)\right] .
\end{aligned}
$$

It is clear from the expressions $(2.35),(2.36)$ that the identity $(2.27)$ holds if $F(\xi)$ is any polynomial in $\xi$ and therefore if $F(\xi)=\exp [-\beta \xi]$.

\section{Classical Heisenberg model as a limit of the quantum Heisenberg model}

Here we prove, in a simple straightforward manner, the results of Millard and Leff [8] and also of Lieb [6] that the free energy of the quantum Heisenberg model, when appropriately scaled, converges to the free energy of the classical Heisenberg model in the large-spin limit. The Lieb method has the advantage over the Millard-Leff approach in that it proves that the thermodynamic free energy converges. The method uses the notion of coherent states and it has been generalised in several directions in the works of Simon [11], Fuller and Lenard [4] and Cesi [2]. The proof presented here is very elementary and does not use the notion of coherent states. Our first result proves convergence for the free energy on a finite box. Let $f_{\mathrm{s}, ~}(\beta, h)$ be the quantum free energy defined as

$$
f_{5,1}(\beta, h)=|\Lambda|^{-1} \log Z_{\mathrm{s}}(\beta, h, \Lambda)-\log (2 s+1)
$$

and $f_{c, 1}(\beta, h)$ be the classical free energy

$$
f_{\mathrm{c}, 1}(\beta, h)=|\Lambda|^{-1} \log Z_{\mathrm{c}}(\beta, h, \Lambda)-\log 4 \pi .
$$

Thus we have $f_{\mathrm{s}, \mathrm{i}}(0,0)=f_{\mathrm{c}, \mathrm{l}}(0,0)=0$.

Theorem 3.1. The following limit holds:

$$
\lim _{s \rightarrow \infty} f_{\mathrm{s.1}}\left(\beta / s^{2}, h s\right)=f_{\mathrm{c}, \mathrm{s}}(\beta, h) \text {. }
$$

Proof. Let $|\boldsymbol{u}\rangle$ be the normalised state defined by (2.11). Then we show that if in $51, \mathrm{~s}$ we replace $h$ by $h s$ we have

$$
\left\langle\boldsymbol{u}\left|\exp \left[-\beta \tilde{S}_{\mathrm{c}}{ }_{1, \mathrm{~s}} / s^{2}\right]\right| \boldsymbol{u}\right\rangle=\int \exp \left[-\beta \tilde{S}_{1, \mathrm{c}}\right] \prod_{R} \frac{1}{2 \pi} \mathrm{d} \theta(R)+\mathrm{O}\left(\frac{1}{\mathrm{~s}}\right)
$$

with $\theta(R)$ defined as in (2.31) and $S_{z}(R)=u_{R} / s-1$. To see this let us suppose we expand out the exponential on the LHS of (3.4) and consider a particular monomial in the $S_{+}(R), S_{-}(R), S_{z}(R), R \in \Lambda$. This will make a non-zero contribution only if for each $R \in \Lambda$ the number of $S_{+}(R)$ in the monomial equals the number of $S_{-}(R)$. Furthermore, when we do any commutation we obtain a $\mathrm{O}(1 / s)$ correction; recall that the operator norms $\left\|S_{x}\right\|,\left\|S_{y}\right\|,\left\|S_{z}\right\| \leqslant s$. Hence we may assume that each monomial is a product of $S_{z}(R)$ and $\frac{1}{2}\left[S_{+}(R) S_{-}(R)+S_{-}(R) S_{+}(R)\right]=s(s+1)-S_{z}(R)^{2}, R \in \Lambda$. The 
sum of all such monomials is from (2.32) precisely the first term on the RHS in (3.4) up to a $\mathrm{O}(1 / \mathrm{s})$ correction.

Next we sum (3.4) over all $\boldsymbol{u}$ with $0 \leqslant u_{R} \leqslant 2 s, R \in \Lambda$, and multiply by $(2 s+1)^{-i . \mid}$. The summation on the $u_{R}$ variable is now

$$
\frac{1}{2 s+1} \sum_{u_{R}=0}^{2 s} \int_{S_{i}(R)=u_{R} / s-1} \frac{1}{2 \pi} \mathrm{d} \theta(R) .
$$

It is evident that this is a Riemann sum for the integration

$$
\frac{1}{4 \pi} \int \mathrm{d} S_{z}(R) \mathrm{d} \theta(R)
$$

which converges as $s \rightarrow \infty$. Thus from (3.4), (3.5), (3.6) we obtain (3.3).

Next we turn to proving the convergence of the thermodynamic free energies. Let $f_{\mathrm{s}}(\beta, h)$ and $f_{\mathrm{c}}(\beta, h)$ be given by

$$
f_{\mathrm{s}}(\beta, h)=\lim _{1 \rightarrow x} f_{\mathrm{s}, 1}(\beta, h) \quad f_{\mathrm{c}}(\beta, h)=\lim _{1 \rightarrow x} f_{\mathrm{c}, \mathrm{s}}(\beta, h) .
$$

It is known [10] that for short-range interactions $J\left(R-R^{\prime}\right)$ the thermodynamic limits (3.7) exist. The theorem we wish to prove in analogy to theorem 3.1 is the following.

Theorem 3.2. The following limit holds:

$$
\lim _{s \rightarrow x} f_{s}\left(\beta / s^{2}, h s\right)=f_{c}(\beta, h) \text {. }
$$

To accomplish this we shall show that the thermodynamic limit (3.7) for the quantum system occurs uniformly in $s$ as $s \rightarrow \infty$. This result taken in conjunction with theorem 3.1 will prove theorem 3.2. For the sake of simplicity we shall assume $J\left(R-R^{\prime}\right)$ is a finite-range interaction but one can extend the method to systems with infinite-range interaction. Thus we shall assume that

$$
\left|J\left(R-R^{\prime}\right)\right| \leqslant J_{0} \quad J\left(R-R^{\prime}\right)=0 \quad \text { if }\left|R-R^{\prime}\right| \geqslant l .
$$

Theorem 3.3. Let $\varepsilon>0$. Then there exists $K_{\varepsilon}(\beta, h)$ depending only on $\varepsilon, \beta, h$ such that for $|\Lambda|>K_{\varepsilon}(\beta, h)$

$$
\sup _{r \geqslant 1}\left|f_{\mathrm{s}}\left(\beta / s^{2}, h s\right)-f_{\mathrm{s}, 1}\left(\beta / s^{2}, h s\right)\right| \leqslant \varepsilon
$$

Proof. Let $\Lambda_{n}$ be a cube with side of length $2^{n}$. Then $\Lambda_{n+1}$ can be divided into eight subcubes each identical to $\Lambda_{n}$. Hence we may write the Heisenberg Hamiltonian on $\Lambda_{n+1}$ as a sum of Heisenberg Hamiltonians on the eight subcubes plus an interaction Hamiltonian $I_{n, s}$. Thus

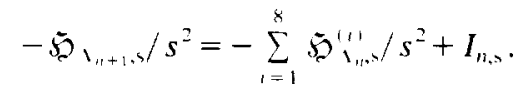

The interaction $I_{n,}$ consists of the interactions between different subcubes and hence from (3.9) we have again using $\left\|S_{\mathrm{r}}\right\|,\left\|S_{y}\right\|,\left\|S_{z}\right\| \leqslant s$ that

$$
-B J_{0} 2^{2 n} l^{3} \leqslant I_{n, \infty} \leqslant B J_{0} 2^{2 n} l^{3}
$$


where $B$ is a universal positive constant which, in particular, is independent of the spin $s$. It follows from a well known trace inequality [10] that

$$
\begin{aligned}
\exp \left[-\beta B J_{0} 2^{2 n} l^{3}\right] & \left\{\operatorname{Tr} \exp \left[-\beta \tilde{S}_{1_{n,},} / s^{2}\right]\right\}^{8} \\
& \leqslant \operatorname{Tr} \exp \left[-\beta \tilde{S}_{1_{n+1 .}} / s^{2}\right] \\
& \leqslant \exp \left[\beta B J_{0} 2^{2 n} l^{3}\right]\left\{\operatorname{Tr} \exp \left[-\beta \tilde{S}_{1_{n,}} / s^{2}\right]\right\}^{8} .
\end{aligned}
$$

Taking the $\log$ of inequality (3.13) and dividing by $\left|\Lambda_{n+1}\right|=2^{3(n+1)}$ we have

$$
\left|f_{s, 1_{n+1}}\left(\beta / s^{2}, h s\right)-f_{\mathrm{s}, 1,(}\left(\beta / s^{2}, h s\right)\right| \leqslant \beta B J_{0} 2^{-n-3} l^{3} .
$$

Since the RHS is summable in $n$ (3.14) clearly implies (3.10).

Proof of theorem 3.2. Choose $\varepsilon>0$ and $|\Lambda|$ large enough so that (3.10) holds and also such that $\left|f_{\mathrm{c}}-f_{\mathrm{c}, 1}\right|<\varepsilon$. For this fixed $\Lambda$ choose $s$ large enough according to theorem 3.1 so that $\left|f_{\mathrm{s} .1}-f_{\mathrm{c} .1}\right|<\varepsilon$. Thus we obtain $\left|f_{\mathrm{s}}-f_{\mathrm{c}}\right|<3 \varepsilon$.

\section{Free gas limit of the Heisenberg model}

Our goal here is to obtain the free Bose gas limit of the quantum Heisenberg model and the Gaussian limit of the classical model. The Bose gas limit for the quantum model corresponds to the magnon approximation. All our results here are proved for positive field $h$. The methods do not apply for $h \rightarrow 0$. Results for $h \rightarrow 0$ have been obtained in [1] for the classical $x-y$ model.

In order to obtain a finite limit we need to normalise the Heisenberg Hamiltonian so that its ground-state energy is zero. Hence for the purposes of this section we define the quantum free energy as

$$
f_{\mathrm{s}, \mathrm{l}}(\beta, h)=|\Lambda|^{-1} \log Z_{\mathrm{s}}(\beta, h, \Lambda)+\beta|\Lambda|^{-1} E_{1,5}
$$

where $E_{1, \mathrm{~s}}$ is the ground-state energy (2.9). The corresponding classical free energy is

$$
f_{\text {c. } 1}(\beta, h)=|\Lambda|^{-1} \log Z_{\mathrm{c}}(\beta, h, \Lambda)+\beta|\Lambda|^{-1} E_{1, \mathrm{c}}
$$

with $E_{\mathrm{1}, \mathrm{c}}$ given by (2.25). We shall again denote the thermodynamic limits of $f_{\mathrm{s}, \mathrm{A}}$ and $f_{c, 1}$ by $f_{\mathrm{s}}, f_{\mathrm{c}}$ respectively.

We first consider the quantum case. To obtain the magnon approximation we drop the quartic terms from Dyson's representation (2.6). This yields a Hamiltonian quadratic in creation and annihilation operators. Finally to evaluate the partition function we replace the restricted trace in (2.17) by the complete trace. The partition function can now be explicitly evaluated. The standard way of doing this is to go into Fourier space. We assume periodic boundary conditions on $\Lambda$ and let $z(R), R \in \Lambda$, be an arbitrary function on $\Lambda$. Then the Fourier transform $\hat{z}(k)$ is given by

$$
\hat{z}(k)=|\Lambda|^{-1 / 2} \sum_{R \in 1} \mathrm{e}^{\mathrm{i} k R} z(R)
$$

where $k$ varies on the dual lattice. Then we have

$$
\sum_{R, R^{\prime} \in 1} \overline{z(R)}\left(\sum_{R} J\left(R-R^{\prime \prime}\right) \delta_{R R^{\prime}}-J\left(R-R^{\prime}\right)\right) z\left(R^{\prime}\right)=\sum_{k} \varepsilon(k)|\hat{z}(k)|^{2}
$$

where

$$
\varepsilon(k)=\sum_{R} J(R)\left(1-\mathrm{e}^{-i k R}\right) .
$$


Thus if $A_{R, R^{\prime}}$ is given by (2.13) then

$$
\sum_{R, R^{\prime} \in 1} a^{*}(R) A_{R R} a\left(R^{\prime}\right)=\sum_{k}(2 s \varepsilon(k)+h) a^{*}(k) a(k)
$$

where the $a(k)$ satisfy canonical commutation relations. Thus the magnon approximation yields

$$
f_{\mathrm{s} .1}(\beta, h) \simeq-|\Lambda|^{-1} \sum_{h} \log \{1-\exp [-\beta(2 s \varepsilon(k)+h)]\} .
$$

We wish to prove a rigorous version of (4.7) in the following.

Theorem 4.1. The following limit holds for $h>0$ :

$$
\lim _{s \rightarrow \infty} f_{s, 1}(\beta / 2 s, h s)=-|\Lambda|^{-1} \sum_{h} \log \{1-\exp [-\beta(\varepsilon(k)+h / 2)]\} .
$$

Proof. Let $\mathcal{N}_{1}$ and $\mathcal{N}_{2}$ be the operators

$$
\hat{N}_{1}=\sum_{R} S_{z}(R)+s \quad \hat{N}_{2}=\sum_{R} a^{*}(R) a(R)
$$

and $\hat{S}_{1, B}$ be the Hamiltonian

$$
\tilde{\mathfrak{Q}}_{\backslash, \beta}=\frac{1}{2} \sum_{R, R^{\prime} \subseteq 1}\left(a^{*}(R)-a^{*}\left(R^{\prime}\right)\right) J\left(R-R^{\prime}\right)\left(a(R)-a\left(R^{\prime}\right)\right)+\frac{b}{2} \sum_{R} a^{*}(R) a(R) .
$$

We put $\mathscr{A}_{1}, \mathscr{A}_{2}$ as

$$
\mathscr{A}_{1}=\exp \left[\frac{-\beta}{2 s}\left(\tilde{S}_{1, \mathrm{~s}}-E_{1, \mathrm{~s}}\right)\right] \quad \mathscr{A}_{2}=\exp \left\{-\beta \tilde{S}_{1, B}\right\} .
$$

Note that the RHS of (4.8) is $|\Lambda|^{-1} \log \operatorname{Tr}\left(\mathscr{A}_{2}\right)$. Using the fact that $\mathscr{N}_{1}$ commutes with $\mathscr{A}_{1}$ and $\mathcal{N}_{2}$ commutes with $\mathscr{A}_{2}$ we have

$$
\begin{aligned}
& f_{\mathrm{s}, 1}(\beta / 2 s, h s)=|\Lambda|^{-1} \log \left(\operatorname{Tr}_{\gamma_{1} \leqslant K} \mathscr{A}_{1}+\operatorname{Tr}_{i,>K} \mathscr{A}_{1}\right) \\
& |\Lambda|^{-1} \log \operatorname{Tr} \mathscr{A}_{2}=|\Lambda|^{-1} \log \left[\operatorname{Tr}_{1: K} \mathscr{A}_{2}+\operatorname{Tr}_{12>K} \mathscr{A}_{2}\right] \text {. }
\end{aligned}
$$

It is easy to see now that since $J \geqslant 0$

$$
\begin{aligned}
\operatorname{Tr}\left(\mathscr{A}_{1}\right) & \leqslant \exp \left[\frac{-\beta h K}{4}\right] \operatorname{Tr} \exp \left[\frac{-\beta h N_{1}}{4}\right] \\
& =\exp \left[\frac{-\beta h K}{4}\right]\left(\frac{1-\exp [-\beta h(2 s+1) / 4]}{1-\exp [-\beta h / 4]}\right)
\end{aligned}
$$

The RHS of (4.13) is uniformly small in $s$ when $K$ is large. Similarly we have

$$
\underset{\mathscr{r}_{2}>K}{\operatorname{Tr}\left(\mathscr{A}_{2}\right)} \leqslant \exp \left[\frac{-\beta h K}{4}\right]\left(1-\exp \left[\frac{-\beta h}{4}\right]\right)^{-|\gamma|}
$$


Next we want to compare $\mathscr{A}_{1}$ and $\mathscr{A}_{2}$ when $\mathcal{N}_{1}, \mathcal{N}_{2} \leqslant K$. Let $\left.\mid m\right)_{R}$ and $|m\rangle_{R}$ be the states defined after $(2.6)$ where $0 \leqslant m \leqslant 2 s$. Then

$$
\begin{aligned}
& \left.\left.a^{*}(R) \mid m\right)_{R}=(m+1)^{1 / 2} \mid m+1\right)_{R} \\
& \left.\left.\left.a(R) \mid m)_{R}=m^{1 / 2} \mid m+1\right)_{R} \quad a^{*}(R) a(R) \mid m\right)_{R}=m \mid m\right)_{R} \\
& \frac{S_{+}(R)}{\sqrt{2 s}}|m\rangle_{R}=(m+1)^{1 / 2}\left(1-\frac{m}{2 s}\right)^{1 / 2}|m+1\rangle_{R} \\
& \frac{S_{-}(R)}{\sqrt{2 s}}|m\rangle_{R}=m^{1 / 2}\left[1+\frac{1-m}{2 s}\right]^{1 / 2}|m-1\rangle_{R} \\
& {\left[S_{z}(R)+s\right]|m\rangle_{R}=m|m\rangle_{R} .}
\end{aligned}
$$

Now if we write

$$
\begin{aligned}
\frac{1}{2 s}\left(\xi_{1, s}-E_{1, s}\right) & \\
= & -\sum_{R, R^{\prime}} \frac{S_{+}(R)}{\sqrt{2 s}} J\left(R-R^{\prime}\right) \frac{S_{-}\left(R^{\prime}\right)}{\sqrt{2 s}}+\sum_{R}\left[h / 2+\sum_{R^{\prime \prime}} J\left(R-R^{\prime \prime}\right)\right]\left(S_{z}(R)+s\right) \\
& -\frac{1}{2 s} \sum_{R, R^{\prime}}\left(S_{z}(R)+s\right) J\left(R-R^{\prime}\right)\left(S_{z}\left(R^{\prime}\right)+s\right)
\end{aligned}
$$

then in view of $(4.15),(4.16)$ we have

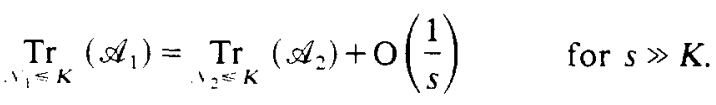

Combining then $(4.13),(4.14)$ with $(4.18)$ the result follows.

Next we wish to prove the thermodynamic results corresponding to (4.8). We shall restrict ourselves to interactions $J\left(R-R^{\prime}\right)$ which are nearest neighbours. Thus

$$
J\left(R-R^{\prime}\right)= \begin{cases}1 & \text { if }\left|R-R^{\prime}\right|=1 \\ 0 & \text { otherwise. }\end{cases}
$$

From (4.5) we see that in this case $\varepsilon(k)$ is given by the formula

$$
\varepsilon(k)=4\left(\sin ^{2} \frac{k_{1}}{2}+\sin ^{2} \frac{k_{2}}{2}+\sin ^{2} \frac{k_{3}}{2}\right)
$$

where $k=\left(k_{1}, k_{2}, k_{3}\right)$.

As $|\Lambda| \rightarrow \infty$ the sum on the right in (4.8) converges to an integral

$$
|\Lambda|^{-1} \sum_{k} \rightarrow \frac{1}{(2 \pi)^{3}} \int_{[-\pi, \pi]^{3}} \text {. }
$$

Theorem 4.2. The following limit holds for $h>0$ :

$\lim _{s \rightarrow \infty} f_{\mathrm{s}}(\beta / 2 s, h s)=\frac{1}{(2 \pi)^{3}} \int_{[-\pi, \pi]^{3}} \mathrm{~d} k \log \{1-\exp [-\beta(\varepsilon(k)+h / 2)]\}$. 
Proof. We shall follow the same strategy as we did in section 3. Let $\Lambda$ be a large cube. Then we have

$f_{s .1}(\beta / 2 s, h s)$

$$
\begin{aligned}
= & |A|^{-1} \log \operatorname{Tr} \exp \left\{\frac { - \beta } { 2 s } \sum _ { R , R ^ { \prime } } \left[\left(S_{-}(R)-S_{+}\left(R^{\prime}\right)\right)\left(S_{-}(R)-S_{-}\left(R^{\prime}\right)\right)\right.\right. \\
& \left.\left.+\left(S_{-}(R)-S_{-}\left(R^{\prime}\right)\right)^{2}\right]-\frac{\beta h}{2} \sum_{R}\left(S_{-}(R)+s\right)\right\}
\end{aligned}
$$

where we sum $R, R^{\prime}$ over each distinct nearest-neighbour pair. It is a well known fact that the thermodynamic limit for $f_{s, 1}$ is independent of boundary condition. Thus we are free to impose periodic, Neumann or Dirichlet conditions on the boundary of $A$. Let us suppose we divide $\Lambda$ into $r$ subcubes $\Lambda, j=1,2, \ldots, r$ each with equal volume. If we impose Neumann (free) boundary conditions on $\Lambda$ and the $\Lambda$, then it is evident that

$$
f_{s, 1}(\beta / 2 s, h s) \leqslant \frac{r\left|\Lambda_{1}\right|}{|\Lambda|} f_{1,1,}(\beta / 2 s, h s)=f_{1,1,}(\beta / 2 s, h s) .
$$

Thus to obtain the inequality (4.22) we have eliminated the bonds across the boundaries $\partial \Lambda_{i}$ of the $\Lambda_{i}, 1 \leqslant i \leqslant r$. Now as in theorem 4.1 we can approximate $f_{\mathrm{s}, 1}$ by the free energy of the free Bose gas. One should note here that, for periodic boundary conditions and Neumann boundary conditions, $\varepsilon(k)$ is given by (4.20). However, $k=\left(k_{1}, k_{2}, k_{3}\right)$ takes different values. In the periodic case $k_{f}=2 \pi n /|\Lambda|^{1 / 3}, n=0,1,2, \ldots,|\Lambda|^{1 / 3}-1$. For the Neumann case $k_{i}=\pi n /|\Lambda|^{1 / 3}, n=0, \ldots,|\Lambda|^{1 / 3}-1$. In either case the sum on the right in (4.8) converges to the integral on the right in (4.22) as $\Lambda \rightarrow \infty$. Hence we have obtained an upper bound on $f$, which is of the correct form.

Next we turn to the lower bound. We obtain this by imposing Dirichlet-type boundary conditions on $\partial \Lambda_{i}, 1 \leqslant i \leqslant r$. For a bond $\left(R, R^{\prime}\right)$ crossing $\partial \Lambda_{i}$ we use the inequality

$\left(S_{+}(R)-S_{+}\left(R^{\prime}\right)\right)\left(S_{-}(R)-S_{-}\left(R^{\prime}\right)\right) \leqslant 2\left(S_{+}(R) S_{-}(R)+S_{+}\left(R^{\prime}\right) S_{-}\left(R^{\prime}\right)\right)$

$\left(S_{z}(R)-S_{z}\left(R^{\prime}\right)\right)^{2} \leqslant 2\left(S_{z}(R)+s\right)^{2}+2\left(S_{z}\left(R^{\prime}\right)+s\right)^{2}$.

Using this inequality to decouple the regions $\Lambda$, we have an inequality

$$
f_{\mathrm{s}, 1}(\beta / 2 s, h s) \geqslant f_{s, 1,1}(\beta / 2 s, h s)
$$

where $f_{5,1}$ has the boundary conditions which agree with (4.25).

Again as in theorem 4.1 we can approximate $f_{4,1}$, by the free energy of a free Bose gas with the same boundary conditions. The free Bose gas Hamiltonian is given by the formula

$$
\begin{aligned}
\mathfrak{S}_{\Lambda_{1}, B}=\sum_{R, R^{*} \in \lambda_{1}}\left(a^{*}(R)-a^{*}\left(R^{\prime}\right)\right)\left(a(R)-a\left(R^{\prime}\right)\right) \\
+\frac{h}{2} \sum_{R} a^{*}(R) a(R)+2 \sum_{R \in \dot{\infty}, 1,} a^{*}(R) a(R)
\end{aligned}
$$

where the sum on $R, R^{\prime}$ is again over all nearest-neighbour pairs. Because of the complicated boundary condition (which is of Dirichlet type but is not exactly a Dirichlet 
condition) it is difficult to diagonalise this Hamiltonian. We therefore compare it to the corresponding periodic Hamiltonian $\mathfrak{S}_{\mathfrak{l}_{1}, B}$ which is easy to diagonalise. Thus

$$
5_{1_{1}, B}^{p}=\sum_{R, R^{\prime} \in 1_{1}}\left(a^{*}(R)-a^{*}\left(R^{\prime}\right)\right)\left(a(R)-a\left(R^{\prime}\right)\right)+\frac{h}{2} \sum_{R} a^{*}(R) a(R)
$$

where we now think of $\Lambda_{1}$ with boundaries identified. Thus we have

$$
\tilde{\mathcal{S}}_{1, B} \leqslant \mathfrak{S \mathcal { Y }}_{1_{1}, B}+2 \sum_{R \in \hat{A} \backslash 1} a^{*}(R) a(R) \text {. }
$$

Consequently we have

$$
\begin{aligned}
& \left|\Lambda_{1}\right|^{-1} \log \operatorname{Tr} \exp \left[-\beta \tilde{S}_{1, B}\right] \\
& \geqslant\left|\Lambda_{1}\right|^{-1} \log \operatorname{Tr} \exp \left(-\beta \bar{S}_{1_{1}, B}^{p}-2 \beta \sum_{R \in \hat{A} \Lambda_{1}} a^{*}(R) a(R)\right) \\
& \geqslant\left|\Lambda_{1}\right|^{-1} \log \operatorname{Tr} \exp \left[-\beta 5 \sum_{1, B}^{n}\right] \\
& -\left|\Lambda_{1}\right|^{-1} \operatorname{Tr}\left(\sum_{R \in \dot{\partial} \Lambda_{1}} a^{*}(R) a(R) \exp \left[-\beta \tilde{S}_{1_{1}, B}^{p}\right]\right)\left\{\operatorname{Tr} \exp \left[-\beta \dot{S}_{1_{1}, B}^{p}\right]\right\}^{-1} \\
& =\left|\Lambda_{1}\right|^{-1} \log \operatorname{Tr} \exp \left[-\beta 5 \mathrm{C}^{p}{ }_{1}, B\right] \\
& -\frac{\beta\left|\partial \Lambda_{1}\right|}{\left|\Lambda_{1}\right|} \operatorname{Tr}\left\{a^{*}(R) a(R) \exp \left[-\beta \tilde{\mathcal{Z}}_{\Lambda_{1}, B}^{p}\right]\right\}\left\{\operatorname{Tr} \exp \left[-\beta \tilde{D}_{1_{1}, B}^{p}\right]\right\}^{-1} .
\end{aligned}
$$

In the inequalities (4.30) we have used the Peierls-Bogoliubov inequality [10] and the translation invariance of the periodic Hamiltonian $5_{L_{1}, B}^{p}$. Since we clearly have

$\operatorname{Tr}\left\{a^{*}(R) a(R) \exp \left[-\beta 5_{S_{1}, B}^{p}\right]\right\}\left\{\operatorname{Tr} \exp \left[-\beta 5_{1_{1}, B}^{p}\right]\right\}^{-1} \leqslant\{1-\exp [-\beta h / 2]\}^{-1}-1$

it follows that by taking $\Lambda_{1}$ sufficiently large that we can approximate as closely as we please the RHS of (4.22). This completes the proof.

The classical version of theorem 4.2 follows in an exactly analogous way to the quantum version. In this case the free energy of the classical Heisenberg model converges to the free energy of a Gaussian model. The Gaussian model is obtained by ignoring the quartic terms in $q(R), p(R)$ in the Dyson Hamiltonian (2.24). For the partition function we integrate $q(R), p(R)$ over all space instead of over the circle of radius 2 as in (2.30). With the same assumptions then as in theorem 4.2 we have the following theorem.

Theorem 4.3. The following limit holds for $h>0$ :

$\lim _{\lambda \rightarrow \infty}\left[f_{c}\left(\lambda^{2} \beta, h / \lambda\right)+2 \log \lambda\right]=\log (\pi / \beta)-\frac{1}{(2 \pi)^{3}} \int_{[-\pi, \pi]^{3}} \log (\varepsilon(k)+h / 2) d k$.

The scaling $\lambda$ on (4.32) is equivalent to replacing integration on the unit sphere in the evaluation of the partition function (1.8) by integration on the sphere of radius $\lambda$.

\section{Free gas upper bounds on the magnetisation}

Here we obtain upper bounds on the magnetisation of the classical and quantum Heisenberg models which correspond respectively to the Gaussian approximation to 
the classical model first discovered by Heller and Kramers [5] and the free Bose gas approximation to the quantum model. The bound for the classical model is an easy consequence of Mermin's theorem [7] although we cannot find it anywhere in the literature. The bound for the quantum model has been proved by Roepstorff [9] in the spin $-\frac{1}{2}$ case. We extend his method to cover all spins.

We consider first the classical case. The magnetisation per unit volume $m$ on the finite cube $\Lambda$ is, from (4.2), given by

$$
m_{\mathrm{c.1}}(\beta, h)=|\Lambda|^{-1} \partial E_{\backslash, \mathrm{c}} / \partial h-\beta^{-1} \partial f_{\mathrm{c}, \mathrm{V}} / \partial h .
$$

If we compute (5.1) from the Gaussian approximation (4.32) for the free energy we see that the Gaussian approximation to the magnetisation is given by

$$
-m_{\mathrm{c}, 1}(\beta, h) \simeq 1-|\Lambda|^{-1} \sum_{k} 1 / 2 \beta(\varepsilon(k)+h / 2) .
$$

We intend to derive a rigorous upper bound on the magnetisation which is given by

$$
\left|m_{\mathrm{c}, 1}(\beta, h)\right| \leqslant\left[1+|\Lambda|^{-1} \sum_{k} 1 / \beta(\varepsilon(k)+h / 2)\right]^{-1 / 2} \text {. }
$$

Evidently when $\beta$ is large the RHS of (5.3) is well approximated by the RHS of (5.2).

To prove (5.3) we use Mermin's theorem [7]. Thus for each $k$ we have the inequality

$$
m^{2} / \beta(\varepsilon(k)+h / 2) \leqslant\left\langle\left|\hat{S}_{+}(k)\right|^{2}\right\rangle
$$

where \langle\rangle denotes the expectation with respect to the classical Heisenberg ensemble. Summing (5.4) over $k$ and using Parseval's theorem we conclude

$$
\begin{aligned}
m^{2}|\Lambda|^{-1} \sum_{k} 1 / & \beta(\varepsilon(k)+h / 2) \leqslant|\Lambda|^{-1} \sum_{k}\left\langle\left|\hat{S}_{+}(k)\right|^{2}\right\rangle \\
= & |\Lambda|^{-1}\left\langle\sum_{R} S_{x}(R)^{2}+S_{y}(R)^{2}\right\rangle=|\Lambda|^{-1}\left\langle\sum_{R} 1-S_{z}(R)^{2}\right\rangle \\
& =1-|\Lambda|^{-1} \sum_{R}\left\langle S_{z}(R)^{2}\right\rangle .
\end{aligned}
$$

Now if we apply Jensen's inequality to see that

$$
m^{2}=\left(|\Lambda|^{-1} \sum_{R}\left\langle S_{z}(R)\right\rangle\right)^{2} \leqslant|\Lambda|^{-1} \sum_{R}\left\langle S_{z}(R)^{2}\right\rangle
$$

then (5.3) follows from (5.5) and (5.6).

Next we turn to the quantum model. Let $m_{5,1}(\beta, h)$ be the magnetisation, where from (4.1)

$$
m_{\mathrm{s}, \mathrm{l}}(\beta, h)=(s|\Lambda|)^{-1} \partial E_{\mathrm{i}, \mathrm{s}} / \partial h-(s \beta)^{-1} \partial f_{\mathrm{s}, \mathrm{l}} / \partial h .
$$

Using the RHS of (4.8) as an approximation to the free energy we have from (5.7) the approximation

$$
-m_{\mathrm{s}, \Lambda}(\beta, h) \simeq 1-(s|\Lambda|)^{-1} \sum_{k}\{\exp [\beta(2 s \varepsilon(k)+h)]-1\}^{-1} .
$$

We prove a rigorous upper bound on the magnetisation which is given by

$$
\left|m_{\mathrm{s}, .}(\beta, h)\right| \leqslant\left(1+(s|\Lambda|)^{-1} \sum_{k}\{\exp [\beta(2 s \varepsilon(k)+h) /|m|]-1\}^{-1}\right)^{-1} .
$$


Note here that $m$ appears on the RHS of (5.9) also. For $\beta$ large it is clear that the RHS of (5.9) is approximately the same as the RHS of (5.8). Inequality (5.9) was obtained by Roepstorff [9] for the case $s=\frac{1}{2}$. Here we extend Roepstorff's method to obtain the inequality for all values of the spin.

The starting point is a quantum version [9] of inequality (5.4). It is given by

$$
s|m| \operatorname{coth}\{\beta(2 s \varepsilon(k)+h) / 2|m|\} \leqslant \frac{1}{2}\left\langle S_{+}(k) S_{-}(k)+S_{-}(k) S_{+}(k)\right\rangle .
$$

One should observe here that if we combine the elementary inequality

$$
x \operatorname{coth} x \geqslant 1
$$

with (5.10) we obtain the Mermin-Wagner inequality [10]. We proceed as previously by summing $(5.10)$ over $k$. Let $I$ be the sum

$$
I=(s|\Lambda|)^{-1} \sum_{k}\{\exp [\beta(2 s \varepsilon(k)+h) /|m|]-1\}^{-1} .
$$

Using the fact that

$$
\operatorname{coth} x=1+2\left(\mathrm{e}^{2 x}-1\right)^{-1}
$$

we have

$$
\begin{aligned}
s|m|(1+2 s I) & \leqslant \frac{1}{2|\Lambda|} \sum_{k}\left\langle S_{+}(k) S_{-}(k)+S_{-}(k) S_{+}(k)\right\rangle \\
& =|\Lambda|^{-1}\left\langle\sum_{R} S_{x}(R)^{2}+S_{y}(R)^{2}\right\rangle \\
& =s(s+1)-|\Lambda|^{-1} \sum_{R}\left\langle S_{z}(R)^{2}\right\rangle .
\end{aligned}
$$

Hence we have the inequality

$$
|m|(1+2 s I)+(s|\Lambda|)^{-1} \sum_{R}\left\langle S_{z}(R)^{2}\right\rangle \leqslant s+1 .
$$

Next we use the translation invariance of the system so we may assume that for any $W \in \Lambda$,

$$
m=s^{-1}\left\langle S_{z}(W)\right\rangle \quad(s|\Lambda|)^{-1} \sum_{R}\left\langle S_{z}(R)^{2}\right\rangle=s^{-1}\left\langle S_{z}(W)^{2}\right\rangle .
$$

Now there are positive numbers $\lambda_{n}>0, n=-s,-s+1, \ldots, s$, such that

$$
\begin{aligned}
& \sum_{n=-s}^{5} \lambda_{n}=1 \\
& \left\langle S_{z}(W)\right\rangle=\sum_{n=-s}^{s} \lambda_{n} n \\
& \left\langle S_{z}(W)^{2}\right\rangle=\sum_{n=-s}^{s} \lambda_{n} n^{2} .
\end{aligned}
$$

From (5.15) we then have

$$
\frac{1}{s} \sum_{n=-s}^{s} \lambda_{n}\left[(1+2 s I) \varepsilon n+n^{2}\right] \leqslant s+1
$$


where $\varepsilon= \pm 1$. Next we choose $\alpha$ to be arbitrary and write

$$
n^{2}=[\alpha+(n-\alpha)]^{2}=\alpha^{2}+2 \alpha(n-\alpha)+(n-\alpha)^{2} .
$$

Thus (5.18) yields

$$
\frac{1}{s} \sum_{n=-s}^{s} \lambda_{n}\left[(1+2 s I) \varepsilon n+\alpha^{2}+2 \alpha(n-\alpha)+(n-\alpha)^{2}\right] \leqslant s+1
$$

which is the same as

$$
(2 \alpha \varepsilon+1+2 s I) \frac{1}{s} \sum_{n=-s}^{s} \lambda_{n} \varepsilon n \leqslant s+1+\frac{\alpha^{2}}{s}-\frac{1}{s} \sum_{n=-s}^{s} \lambda_{n}(n-\alpha)^{2} .
$$

Next we take $\alpha \varepsilon=s-\frac{1}{2}$ which yields

$$
2(1+I) \sum_{n=-s}^{5} \lambda_{n} \varepsilon n \leqslant 2 s+\frac{1}{4 s}-\frac{1}{s} \sum_{n=-s}^{s} \lambda_{n}(n-\alpha)^{2} .
$$

Since $|n-\alpha| \geqslant \frac{1}{2}$ we obtain the inequality

$$
\frac{1}{s} \sum_{n=-\varsigma}^{s} \lambda_{n} \varepsilon n \leqslant(1+I)^{-1}
$$

and this is just the inequality (5.9) on taking $\varepsilon= \pm 1$.

\section{Acknowledgments}

We would like to thank Paul Federbush for helpful conversations. This work was partially supported by the US National Science Foundation grant DMS 8900244.

\section{References}

[1] Bricmont J, Fontaine J, Lebowitz J, Lieb E and Spencer T 1981 Lattice systems with a continuous symmetry Commun. Math. Phys. 78 545-66

[2] Cesi F 1989 D-component rotators as classical limit of Quantum $\mathrm{SO}(\mathrm{D})$ vector models Preprint Courant Institute

[3] Dyson F 1956 General theory of spin-wave interactions Phys. Rev. 102 1217-30

[4] Fuller W and Lenard A 1979 Generalised quantum spins, coherent states, and Lieb inequalities Commun. Math. Phys. 67 69-84

[5] Heller $G$ and Kramers $H 1956$ Ein klassisches Modell des Ferromagnetikums und seine nachtragliche quantisierung in Gebiete tiefe temperaturen $H$ A Kramers Collected Scientific Papers (Amsterdam: North-Holland) pp 598-606

[6] Lieb E 1973 The classical limit of quantum spin systems Commun. Math. Phys. 31 327-40

[7] Mermin N 1967 Absence of ordering in certain classical systems J. Math. Phys. 8 1061-4

[8] Millard K and Leff $\mathrm{H} 1971$ Infinite spin limit of the quantum Heisenberg model J. Math. Phys. 12 1000-5

[9] Roepstorff G 1977 A stronger version of Bogoliubov's inequality and the Heisenberg model Commun. Math. Phys. 53 143-50

[10] Ruelle D 1969 Statistical Mechanics: Rigorous Results (New York: Benjamin)

[11] Simon B 1980 The classical limit of quantum partition functions Commun. Math. Phys. 71 247-76 